\title{
Commentary
}

\section{What ME/CFS caregivers want you to know}

\author{
Bobbi Ausubel \\ Caregiver and Advocate, Walnut Creek, CA, USA \\ E-mail: bobbi.gloria.ausubel@gmail.com
}

Received 10 December 2019

Accepted 7 February 2020

Eight stories comprise this paper: Stories were written in 2019 from caregivers, parents, and spouses, most of whom live with a pwME (person with ME). To protect those in our care, some authors have not used their full names. Each patient suffers from Myalgic Encephalomyelitis/Chronic Fatigue Syndrome (ME/CFS), a neuro-immune disease which affects each person differently, with a great variety of symptoms and a wide spectrum of disease burden. The National Academy of Medicine says ME/CFS is three times more common in women than men [1], and they estimate that $80 \%$ of patients have not yet received a diagnosis [2]. Through their experiences caring for loved ones with ME/CFS, these caregivers have anecdotes and requests for future doctors and occupational therapists to assist with servicing their suffering pwMEs.

$* * * * * * * * * * * * * * * * * * * * * * * * * * * * * * * * * * * * * * * *$

\section{J., Mother and at-home caregiver of a $p w M E$}

My brilliant and beautiful daughter was stricken with ME/CFS in college. Ten years later, she is mostly homebound, suffering every hour of every day. As her caretaker, I must do all the shopping, cooking, cleaning, and laundry. I must help her with showering and dressing.

My daughter has lost everything to this disease... a career, friends, family gatherings, and so much more - even going outside. My own career has suffered as so much time is needed to care for her.

She has multiple severe chemical sensitivities. Just last week we had a visiting nurse come. Prior to coming to the appointment, the nurse was asked to use no fragrance, no hair spray, deodorant, etc., but upon her arrival it quickly became apparent that she had not adhered to this request. When the nurse walked in, my daughter began to vomit. The nurse had washed her clothes in Tide. This gives you an idea about how sensitive patients are to so many things. Going to a doctor's office with its smells and noise and lights can send my daughter into a relapse.

It would be great to work with an occupational therapist (OT) to assess our home and make suggestions about the ways in which my daughter's activities could be less stressful. For example, I recently bought a faucet that can be turned on by tapping it anywhere. This saves my daughter from the pain of reaching for the handles. ME/CFS is a disease of energy depletion. There are probably many ways an OT could make tasks easier.

Thank goodness she has an understanding doctor. Though he cannot make her better, he is kind and compassionate and that makes a world of difference.

$* * * * * * * * * * * * * * * * * * * * * * * * * * * * * * * * * * * * * * * *$

Courtney Miller, at-home caregiver and wife of Robert Miller, an ME/CFS patient of 30 years

The love of my life has Myalgic Encephalomyelitis (ME/CFS).

That meant holding a mirror under my husband's nose in the early days to make sure he was still breathing, after days or weeks of coma-like sleep. It meant seeing dozens of doctors for a diagnosis and searching for years to find a doctor who had a plan - not a cure, but a plan. Compassionate providers who listen make all the difference.

It meant watching our 9-year old sons cry as they left the only friends they really knew, as we moved 
back to Reno to access the only treatment in an FDAapproved clinical trial, an immune modulator called Ampligen. I knew Ampligen would save their dad's life, which it did.

There are still no FDA-approved treatments for the disease. The treatment lessened his pain enough to allow him to be out of bed when the boys got home from school, and they could watch Dolphins games together.

Scientists hold the power and the promise of identifying treatments that can make my husband and more than 2 million patients' lives shine. Caregiving is more than taking him to doctor's visits or the emergency room. It was giving him my hope when he couldn't find his, and my determination when his needed company. But the most important care to give was helping him pursue the lone treatment trial for his disease because that made everything else possible.

***********************************************

Elizabeth B. Burlingame, mother and at-home caregiver of a pwME

When a health assistant comes to my home, before they treat my daughter, Elizabeth Ann Burlingame, I would like them to know that for 28 years, she has been disabled. Prior to that time, she was an active healthy young lady aspiring to save the environment.

Then she came down with something like the flu. She never recovered. We saw over 20 doctors and found that she has Chronic Fatigue Syndrome.

There is now a new name, ME/CFS, but still no treatment or cure.

She is now housebound (mostly bedbound). Her day begins with a cup of tea around midmorning as she moves slowly. I bring her up a tray and she eats small amounts of healthy foods. Her digestive tract is extremely sensitive. She deals with body aches and uses cold packs around her head to try and relieve some of the pain. She deals with cognitive issues at her worst moments. If she tries to enjoy having a friend or family member visit, she will pay dearly. Her symptoms all become worse.

We have exhausted all medical support we can and try to support awareness through advocacy work.

$* * * * * * * * * * * * * * * * * * * * * * * * * * * * * * * * * * * * * * * * *$

Katy C., mother of a pwME

As a parent, the beginning of this journey felt as if our child was slipping through our fingers. January of junior year of high school, after a normal holiday illness, she realized she couldn't think. She would walk away not realizing a friend was in the middle of a sentence. She could hear a class lecture, but it made no sense. By April, her exhaustion was so profound that she struggled to walk from the car to class. I picked her up every day at lunch; she was ashen by then. We kept thinking she would get better. She was a former club soccer player, a top student. This didn't make sense.

From 2010-2017, we went through tests run by rheumatologists, pediatric neurologists, allergists, ENT's, PsycD's, etc. Nothing was wrong. But something was very clearly wrong. We were lucky to have very collaborative doctors, although it was hard for them to sense the level of sheer exhaustion through which she struggled. Fortunately, she would get red, hot rashes on her joints, knees, and calves, because otherwise she worried that maybe she was making this up.

In 2017, we finally we landed with an infectious disease specialist whose son had been ill for years. $\mathrm{He}$ understood the battle. Our daughter's blood test revealed Coxsackie B antibodies as high as the test could register. We were relieved. Here was something! Or maybe it was something. The most difficult thing about a disease without a clear set of diagnostic parameters is that you're never really sure what it is, or how to treat it. But we were with someone who was determined to make the journey with us.

She is halfway through a $\mathrm{PhD}$ and, although she can seldom be out with friends, she is grateful that she functions as well as she does. Some people have been fighting this illness for thirty years. There are people who are bedridden, who can't stand light or sound. It is terrifying. With NIH recognition of ME/CFS, we feel like we are gaining momentum among the medical community. We can be on this voyage together, patients, clinicians, researchers ... and parents, who never lose hope that there is a way for their child to win back their future.

If healthcare professionals would let people with ME know that exercise can make them worse, and if they could help patients understand that relative inactivity is a guard against further decline - it would be helpful. Jen Brea, who was a Harvard grad student when she fell ill with ME, wishes she had understood this herself. Her Sundance award-winning documentary, Unrest, is available on Netflix. It's riveting and a great primer. 
Our daughter says that the exhaustion and brain fog make processing information difficult. Slowing down discussions in general, and repeating information if someone looks a little lost would be great. No one really likes to admit that information has bypassed them.

***********************************************

\section{Bev., mother of a pwME}

Before you walk in the door to treat my daughter, try to understand what we I have gone through.

She became ill about 14 years ago and was not diagnosed until ten years later. We did what everyone else does; went from doctor to doctor to no avail. She has been home-bound for two years. She worked for the same company for 18 years, initially in the office, then telecommuted full-time. Two years ago, her condition worsened. The company allowed her to work part-time, but she became so ill that she was unable to return to work when required. She lost her job.

With her wishes and long-term hopes diminished, her goal is "to be well enough to work part-time." She no longer dreams of vacations or having a family. She is gifted and talented, like so many of the other incredible ME/CFS patients I encountered in my advocacy work, but she is also isolated, surrounded by four walls and dependent on the computer/phone for human connection.

I am not her hands-on caregiver. She is married. I help however I can; she knows I am there for her.

If I think about it, it is almost too much. I am consumed by sadness for my 40+-year old child. How do you help this pwME, what can you say? I began to see a therapist to learn how to be emotionally supportive, to learn how to tolerate the unbearable. I volunteer and advocate unrelentingly.

There are fewer than two dozen ME/CFS specialists across the US. My daughter in California is fortunate now; she only has to travel 60 miles each way to see hers.

Estimating from the National Academy of Medicine report there are up to 300,000 Californians with ME/CFS [3], an estimated 84 to 91 percent of whom are still undiagnosed or misdiagnosed [4].

So, as a mother, I ask you to imagine that you have a child/spouse who is unable to leave their home, or worse, their bed. Unable to work, with so much potential that cannot be used. Then, treat my daughter compassionately.

*******************************************************

\section{Martin W., father of a pwME}

Cancer, heart disease, HIV/AIDS, Parkinson's. All are horrific diseases. But those afflicted can find a doctor or medical center for treatment, usually near their home. This is not the case for the more than $100,000+$ persons in California with ME/CFS, 25 percent of whom are bed-bound, many unable to tolerate sound or light.

Many cannot work and are homebound, as is my daughter. Many are misdiagnosed and go from doctor to doctor to no avail.

We ask you if you treat our daughter:

- Do not advise a pwME to do physical exercises; they only make patients more ill.

- See the award-winning, free documentary, Unrest (available on Netflix) to better understand ME/CFS.

- Take other courses about ME to get CME credits. There are credits available for watching Unrest at https://www.unrest.film/cme and other places.

- Check out: Medscape: Diagnosing ME/CFS:

The Experts Weigh In, https://www.medscape. org/viewarticle/907632_3.

- Michigan State Medical Society: Update on Chronic Fatigue Syndrome Part 1: Clinical Diagnostic Criteria for Chronic Fatigue Syndrome/CFS now called Myalgic Encephalomyelitis or ME/CFS.

- Update on Chronic Fatigue Syndrome Part 2: Uniting Compassion, Attention, and Innovation to Treat ME/CFS, https://connect.msms.org/Education-Events/On-Demand-Webinars?topic $=2$.

- Medridge Education: ME/CFS Part 1: Introduction and Identification: ME/CFS Part 2: Etiology and Analeptic Management, https://www.medbridgeeducation.com/courses/details/me-cfs-part-1-introduction-identification-todd-davenportstaci-stevens-mark-van-ness?fbclid=IwAR3NP8 KoQA-4QtGBUE_mZ5q0nr68yRFmZBdBd5w E6sxYs5N8W7GnY9inH7I.

$* * * * * * * * * * * * * * * * * * * * * * * * * * * * * * * * * * * * * * * * * *$

\section{B., mother of a $p w M E$}

My daughter has been ill with Myalgic Encephalomyelitis/Chronic Fatigue Syndrome (ME/CFS) for 30 horrible years.

My daughter cannot receive visits from health workers or state-funded cleaning persons because she has multiple chemical sensitivities, one of the 
many symptoms that pwMEs experience. Helpers have used perfumed shampoo, soap, or laundry detergent, or carry odors from their homes or cars that make my daughter sicker. Finding a home for her was an awful experience. We walked into one vacant apartment, looked around, came out, and she threw up immediately because of the chemicals, maybe petroleum from candles or cleaning products. The gas station next door was also an issue.

Making and keeping medical appointments is hard because there are days when she is so weak, and changes in in energy levels that may cause the pwME to miss appointments. Sitting in an office waiting to be seen can be treacherous. So many fragrances and stimuli abound there, making visits painful. Sadly, the exhaustion of traveling and being in the waiting room can produce more suffering than the needed visit is worth.

There is also cognitive impairment. She is often overwhelmed by even small amounts of information, and she cannot retain it. All information needs to be written down for her.

She has experienced occasional paralysis recently. My daughter wonders if rapid heartbeat and difficulty breathing might cause her to die. Patients have died. Significantly, she personally knows some of those who have died. A cloud hovers around us-we are all aware that lack of hope and harshness of symptoms have caused suicides. PwME's help each other online to 'hold on'. And since most doctors do not have training in diagnosing ME/CFS, the treatments at hospitals are more often harmful than helpful.

Kindness is the most important need. Feeling support is healing all by itself; the health care professional who listens and truly wants to understand $\mathrm{ME} / \mathrm{CFS}$ is a healing gift.

**************************************

M' Liz C., friend of two pwME

I have watched my friends struggle with the aftermath of an ME diagnosis.

I learned about ME from a good friend whose daughter became ill 10 years ago. I tried to be a supportive friend, watching her search for an effective treatment, without success. Then, just last year, another friend's daughter was also diagnosed with ME, shortly after becoming engaged. I watched this daughter struggle with her daily routine, unable to work, during what should have been the happiest time of her life, terrified that she would go down the aisle in a wheelchair. She then paid the price for her day of happiness when she spent two months after her wedding in the hospital, half of those in intensive care. Her doctors have struggled to help her, but her future is uncertain at best, her ability to start a family unclear.

I recently met a nurse named Jane Pannell who worked at the University of California/San Francisco Hospital (UCSF) for 20 years. A hardworking clinician and researcher, she became the Project Director for a national HIV research program. Diagnosed with ME in 1984, her disease progressed until she was unable to work, and she sought care from UC. UC was unable and unwilling to help her. Frustrated, she feels UC has let her down in her time of need.

My main advice: There is something you can do, please help people like my friends' daughters and Jane to regain their lives by diagnosing and treating those with ME with compassionate and informed care.

\section{Conflict of interest}

None to report.

\section{References}

[1] National Academy of Medicine, National Academy of Sciences (2015). Beyond Myalgic Encephalomyelitis/Chronic Fatigue Syndrome: Redefining an Illness. The National Academies Press, Washington, DC: 2015, P243

[2] National Academy of Medicine, National Academy of Sciences (2015). Beyond Myalgic Encephalomyelitis/Chronic Fatigue Syndrome: Redefining an Illness. The National Academies Press, Washington, DC: 2015, P1

[3] Estimated from: National Academy of Medicine, National Academy of Sciences (2015). Beyond Myalgic Encephalomyelitis/Chronic Fatigue Syndrome: Redefining an Illness. The National Academies Press, Washington, DC: 2015, P1 Note: The CA estimate of up to 300000 comes from using the $2.5 \mathrm{M}$ upper limit in the NAM report (page 1) and multiplying by the fraction of Americans who live in CA.

[4] National Academy of Medicine, National Academy of Sciences (2015). Beyond Myalgic Encephalomyelitis/Chronic Fatigue Syndrome: Redefining an Illness. The National Academies Press, Washington, DC: 2015 P1 Note: The CA estimate of up to 300000 comes from using the $2.5 \mathrm{M}$ upper limit in the NAM report (page 1) and multiplying by the fraction of Americans who live in CA. 